Coagulation abnormalities, bleeding, thrombosis, and management of patients with acute liver failure in Australia and New Zealand

\author{
Stephen Warrillow ${ }^{1,2}$ \\ Caleb Fisher ${ }^{1}$ \\ Heath Tibballs ${ }^{1}$ \\ Michael Bailey ${ }^{2,3}$ \\ Colin McArthur ${ }^{4,5}$ \\ Pia Lawson-Smith ${ }^{5}$ \\ Bheemasenachar Prasad ${ }^{6}$ \\ Matthew Anstey ${ }^{7}$ \\ Bala Venkatesh ${ }^{8}$ \\ Gemma Dashwood ${ }^{8}$ \\ James Walsham ${ }^{8}$ \\ Andrew Holt ${ }^{9}$ \\ Ubbo Wiersema $^{9}$ \\ David Gattas ${ }^{10}$ \\ Matthew Zoeller ${ }^{10}$ \\ Mercedes Garcia Alvarez ${ }^{11}$ \\ Rinaldo Bellomo $1,2,12,13,14$
}

1. Department of Intensive Care, Austin Health, Melbourne Australia

2. Department of Medicine and Surgery, The University of Melbourne, Melbourne, Australia

3. Australian and New Zealand Intensive Care Research Centre, Monash University School of Public Health and Preventive Medicine, Melbourne Australia

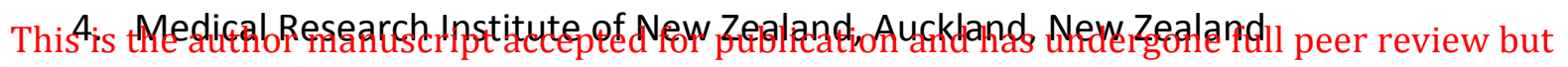

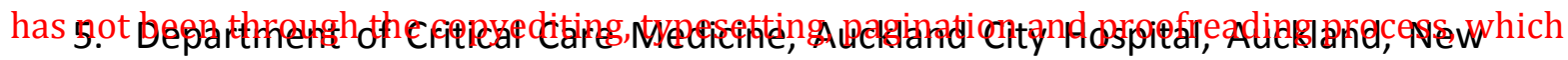
may lead to differences between this version and the Version of Record. Please cite this article as doi: Ze.1111/jgh.14876

This article is protected by copyright. All rights reserved. 
6. South Metropolitan Health Service, Rockingham, Australia

7. Department of Intensive Care, Sir Charles Gairdner Hospital, Perth, Australia

8. Department of Intensive Care, Princess Alexandra Hospital, Brisbane, Australia

9. Department of Intensive Care, Flinders Medical Centre, Adelaide, Australia

10. Department of Intensive Care, Royal Prince Alfred Hospital, Sydney, Australia

11. Department of Anesthesiology and Pain Medicine, Hospital Santa Creu i Sant Pau, University of Barcelona, Spain

12. Department of Intensive Care, Royal Melbourne Hospital, Melbourne, Australia

13. Data Analytics Research and Evaluation (DARE) Centre, Austin Hospital and University of Melbourne, Melbourne, Australia

14. 14. Centre for Integrated Critical Care, The University of Melbourne, Melbourne, Australia

For the Australasian Management of Acute Liver Failure Investigators (AMALFI)

Corresponding author:

Stephen Warrillow

Department of Intensive Care, Austin Health

145 Studley Road, Heidelberg Victoria 3084 Australia

Stephen.Warrillow@austin.org.au

This research did not receive any specific grant from funding agencies in the public, commercial, or not-for-profit sectors. None of the authors have any relevant conflicts of interest to declare.

This article is protected by copyright. All rights reserved. 


\title{
Coagulation abnormalities, bleeding, thrombosis, and management of patients with acute liver failure in Australia and New Zealand
}

\begin{abstract}
Background and Aim: To study the management of coagulation and hematological derangements among severe acute liver failure (ALF) patients in Australia and New Zealand (ANZ) liver transplant Intensive Care Units (ICUs).

Methods: Analysis of key baseline characteristics, etiology, coagulation and hematological tests, use of blood products, thrombotic complications and clinical outcomes during the first ICU week.

Results: We studied 62 ALF patients. The first day median peak INR was 5.5 (IQR 3.88.7), median longest aPTT was 62 seconds (IQR 44-87) and median lowest fibrinogen was 1.1 (IQR 0.8-1.6) g/L. Fibrinogen was only measured in $85 \%$ of patients, which was less than other tests $(p<0.0001)$. Median initial lowest platelet count was 83 (IQR 41122) $\times 10^{9} / \mathrm{L}$. Overall, $58 \%$ of patients received fresh frozen plasma, $40 \%$ cryoprecipitate, $35 \%$ platelets and $15 \%$ prothrombin complex concentrate. Patients with bleeding complications (19\%) had more severe overall hypofibrinogenemia and thrombocytopenia. Thrombotic complications were less common (10\% of patients), were not associated with consistent patterns of abnormal hemostasis, were not immediately preceded by clotting factor administration and half occurred only after liver transplantation surgery.

Conclusion: In ALF patients admitted to dedicated ANZ ICUs, fibrinogen was measured less frequently than other coagulation parameters but, together with platelets, appeared more relevant to bleeding risk. Blood products and pro-coagulant factors were administered to most patients at variable levels of hemostatic derangement, and bleeding complications were more common than thrombotic complications. This epidemiologic information and practice variability provide baseline data for the design and powering of interventional studies.
\end{abstract}

This article is protected by copyright. All rights reserved. 
Key words: Acute liver failure, coagulopathy, bleeding, thrombosis, encephalopathy emergency liver transplant

This article is protected by copyright. All rights reserved. 


\section{Introduction}

Acute liver failure (ALF) leading to ICU admission carries a high mortality ${ }^{1}$ and more than half of all cases in ANZ are due to paracetamol overdose (POD) ${ }^{2}$. Regardless of etiology, loss of liver function results in a characteristic pattern of illness, including abnormal measures of hemostasis that are the hallmarks of $\mathrm{ALF}^{3}$. These include elevation of the international normalized ratio (INR) or prolongation of the prothrombin time ${ }^{4}$, with such changes often being a focus of treatment ${ }^{5}$. Despite being a defining characteristic of ALF, little is known about how changes in INR and other common tests of hemostasis change over time and relate to outcome and bleeding complications, or how clinicians respond by administering clotting factors of blood products and how such administration affects the risk of thrombosis., 7

Accordingly, we conducted an exploratory evaluation of hemostatic abnormalities and their current ICU management in ANZ. We hypothesized that different markers of hemostasis might be variably monitored and yet may be associated with higher risk of bleeding complications. We further hypothesized that there would be complex patterns of blood product and clotting factor utilization with considerable practice variation. Finally, we hypothesized that bleeding complications would be more common than thrombotic complications.

\section{Methods}

\section{Study design}

As previously described ${ }^{2}$, all adult liver transplant ICUs in ANZ hospitals were invited to submit detailed de-identified retrospective clinical data relating to adult patients admitted to ICU with ALF over a four year period concluding at the end of 2016, using a standardized collection tool. Local requirements governing data sharing were adhered to at each site. Austin Health Ethics committee approval was obtained (LNR/14/Austin/676) and, depending on local requirements, either a Memorandum of 
Understanding (MoU), or a Clinical Trials Research Agreement (CTRA) was implemented.

Information for each patient was obtained from the clinical record and only included routinely documented usual patient care related data. Each ICU submitted ten or more consecutive patients coded as ALF (APACHE III diagnostic code 301.01), with onset of overt liver failure over a period of less than eight weeks in the absence of known preexisting liver disease.

Variables collected included background characteristics, cause of ALF, illness severity, components of the Kings College Criteria (KCC), pathology test results, interventions, blood product utilization and key outcomes. Data were collected at ICU admission, 6, 12, and 24 hours after admission and then every day for one week. A sample size of approximately 60 patients was deemed sufficient on the basis of convenience and feasibility. The selection of at least ten consecutive patients from each site, regardless of outcome or etiology of ALF, was undertaken to achieve balanced representation across the region. Bleeding episodes were defined as documented instances of any hemorrhage requiring intervention or specific imaging with or without a subsequent blood transfusion. Thrombotic complications evaluated for included any venous thrombosis (including portal vein thrombosis), pulmonary embolism, acute myocardial infarction, ischemic stroke and any arterial thrombosis. To assess the relationship between bleeding complications and hemostatic parameters, two exploratory models were developed. The first model evaluated differences between hemostatic parameters recorded at around the time of bleeding versus those from the same patient documented at a randomly selected, temporally distant period without documented active bleeding. The second model, at a similar length of time from ICU admission, compared hemostatic parameters from around the time of a bleeding episode in those patients who bled to those in matched patients who did not (matching was according to age ( \pm 5 years), ALF etiology and KCC status). A similar 
approach was also used to explore the relationship between thrombotic complications and hemostatic parameters. The design and reporting of this study was aligned with the Strengthening the Reporting of Observational Studies in Epidemiology (STROBE) recommendations ${ }^{8}$.

\section{Statistical analysis}

Statistical analysis was performed using IBM SPSS statistics for Macintosh, version 25 (IBM Corporation, Armonk, NY, USA). All assessments and comparisons relate to data collected in the first week in ICU. Continuous variables are expressed as medians with inter-quartile range (IQR), whilst categorical variables as frequencies with percentages. Continuous data were compared using the Mann-Whitney test. Categorical data were compared using Chi Square or Fisher's exact test as appropriate. The Wilcoxon signedrank test was used for comparisons of tests taken at different times in the same patients and matched patients for the exploratory model. Longitudinal data was assessed for normality and log-transformed where appropriate. Differences between groups over time were analyzed using repeat measures analysis of variance fitting main effects for group, time and an interaction between group and time to determine if groups behaved differently over time. These results are present as means with standard error or as geometric means with $95 \%$ confidence interval $(95 \% \mathrm{Cl})$ in accordance with the underlying distribution of the data. To correct for multiplicity of comparisons, a two-sided $p$-value of $<0.01$ was used to indicate statistical significance.

\section{Results}

Patient characteristics, etiology of ALF and clinical outcomes

We studied 62 ALF patients (see supplementary appendix Table 1). Just over half of cases were due to POD, 60\% fulfilled King's College Criteria (KCC) for transplantation and $20 \%$ overall underwent emergency liver transplantation (ELT). Nearly a third of patients died, with all deaths occurring in the ICU. Only half achieved ELT-free survival. Intensity of testing of coagulation and hematological variables

This article is protected by copyright. All rights reserved. 
Coagulation and hematological variables were monitored in most patients at the defined times (Table 1). However, fibrinogen, was measured less often than INR $(p<0.0001)$ and was not checked at all in $9(15 \%)$ patients.

International Normalized Ratio (INR)

The INR was elevated on admission in every patient (Figure. 1). The mean initial peak INR was 5.5 (IQR 3.8-8.7) with no difference between patients with or without subsequent bleeding complications. During the remaining week of ICU management, patients with a bleeding episode had an overall mean INR of 3.0 (95\% Cl 2.3-3.7) compared with $2.3(95 \% \mathrm{Cl} 2.0-2.5)$ for those without bleeding $(p=0.02)$ (supplementary appendix Figure 1).

Activated Partial Thromboplastin Time (aPTT)

Overall, 47 (76\%) patients had a prolonged aPTT on day 1, with a mean longest aPTT of 62 seconds (IQR 44-77). The aPTT shortened to within the normal range after this time, varying little thereafter (Figure 1). There was no overall significant difference in aPTT according to bleeding complications (supplementary appendix Figure 2).

Fibrinogen

At ICU admission, $77 \%$ of all fibrinogen results were abnormally low, with a median lowest initial concentration of $1.1 \mathrm{~g} / \mathrm{L}$ (IQR 0.8-1.6). The fibrinogen concentration remained persistently low throughout ICU admission (Figure 1). Compared with patients without a bleeding episode those with a bleeding complication had worse overall hypofibrinogenemia in ICU (mean $1.1 \mathrm{~g} / \mathrm{L}(95 \% \mathrm{Cl} 0.8-1.4)$ versus $1.8 \mathrm{~g} / \mathrm{L}(95 \% \mathrm{Cl}$ 1.6-2.0); $p=0.0004$ ) (Figure 2).

\section{Platelet count}

The median initial lowest platelet count was $83 \times 10^{9} / \mathrm{L}$ (IQR 41-122) and 53 (85\%) patients had thrombocytopenia. The median platelet count decreased over the next two days and then stabilized (Figure 1). Patients who experienced an episode of bleeding had a significantly lower nadir count on day one (Table 2) and more severe thrombocytopenia overall whilst in ICU (mean $44 \times 10^{9} / \mathrm{L}(95 \% \mathrm{CI} 32-60)$ versus $89 \times$ $10^{9} / \mathrm{L}(95 \% \mathrm{Cl}$ 76-105); $\mathrm{p}<0.0001)$ (Figure. 3).

This article is protected by copyright. All rights reserved. 
Blood product use

Fresh Frozen Plasma (FFP)

Overall, 36 (58\%) patients received FFP with $89 \%$ receiving their first dose on day one. Few patients received FFP after day three (supplementary appendix Figure 3). For FFPtreated patients, the median total dose was 5.5 units (IQR 4.0-10.0). The peak INR on day 1 for FFP-treated patients was 5.7 (IQR 4.1-9.2) compared with 5.0 (IQR 3.4-7.0); $\mathrm{p}=0.21$ ) for FFP-untreated patients. Patients with a bleeding episode were more likely to receive FFP (Table 2); however, $50 \%$ of patients without bleeding also received FFP. Cryoprecipitate (CRYO)

Overall, 25 (40\%) patients received cryoprecipitate (CRYO) with 76\% receiving their first dose on day one. For CRYO-treated patients, the median total dose was 16 units (IQR 10-30 units). Their lowest fibrinogen on day one was $0.9 \mathrm{~g} / \mathrm{L}$ (IQR 0.8-1.1) (supplementary appendix Figure 4), compared with $1.2 \mathrm{~g} / \mathrm{L}$ (IQR 0.8-1.6) for CRYOuntreated patients $(p=0.001)$. Patients with a bleeding episode were more likely to receive CRYO (Table 2). However, $32 \%$ of patients without bleeding also received CRYO.

\section{Prothrombin Complex Concentrate (PCC)}

Only 9 (15\%) patients received three factor PCC (four factor PCC is unavailable in ANZ). Eight received their first dose on day one and $71 \%$ of the overall amount was given by then. For PCC-treated patients, the median dose was 2500 units (IQR 2000-4000). The median peak INR on day one for PCC-treated patients was 10.1 (IQR 5.4-10.7) vs. 5.1 (IQR 3.8-7.0) for untreated patients $(p=0.02)$. All PCC-treated patients fulfilled KCC (24\% (9/37); $p=0.001)$.

\section{Recombinant Factor VII-activated (rFVIIa)}

Only 4 (6\%) patients received recombinant Factor VII-activated (rFVIla.) All were cared for in the same ICU and treatment occurred exclusively on day one, with a median total dose of $5.2 \mathrm{mg}$ (IQR 4.8-5.6). No patient experienced a bleeding episode or

This article is protected by copyright. All rights reserved. 
thrombotic complication, and none achieved ELT-free survival, with two dying and two surviving after ELT.

\section{Platelet transfusion}

Overall, 22 (35\%) patients received at least one platelet transfusion, with 64\% receiving their first transfusion on day one. For platelet-transfused patients, the median number of pooled bags (each bag containing four units) transfused during the first week was 2.5 (IQR 1-4). The median nadir platelet count on the first day for transfused patients was $44 \times 10^{9} /$ I (IQR 35-96) (supplementary appendix Figure 5), vs. $102 \times 10^{9} /$ I (IQR 72-140), for non-transfused patients $(p=0.004)$. Platelets were given for 11 of 12 bleeding episodes, with $76 \%$ of all administered platelet given at this time. Packed Red Blood Cells (PRBC)

Overall, 29 (47\%) patients received a blood transfusion, with a median amount of 3 units (IQR 1-4 units). The median nadir hemoglobin concentration on day one for transfused patients was 80g/L (IQR (40-141) vs. 111 g/L (IQR 102-123) for nontransfused patients $(p<0.001)$. Numerically, more KCC patients were transfused $(59 \%$ vs. $28 \%$; $p=0.02$ ). Patients who bled were also more likely to be transfused (Table 2 ).

\section{Bleeding complications}

Twelve patients had a bleeding complication (Table 2). These patients were younger and appeared more severely ill. The median time from ICU admission to the occurrence of the bleeding episode was 12 hours (IQR 6-161). Two patients received FFP at some point during the 24-hour period preceding the onset of their hemorrhage. Diffuse upper gastrointestinal oozing was the commonest form of bleeding (five patients). However, only one of these patients underwent endoscopic intervention. Bleeding from vascular access sites affected three patients, however, this was only associated with the actual line insertion procedure in one patient. None required specific interventions other than blood product administration. Bleeding occurred at the site of recent non-ELT related major surgery in two patients and necessitated further operative intervention, while one other patient had a spontaneous intra- 
abdominal hemorrhage. Spontaneous intracranial bleeding occurred in two patients. No significant differences in the requirement for critical care supports was evident except for mechanical ventilation, which was used more often in patients with a documented bleeding episode (12/12 bleeding patients versus 35/50 non-bleeding patients; $\mathrm{p}=0.02$ ). Management and outcome details are shown in supplementary appendix Table 2.

In multivariable models, a longer recent aPTT and a lower platelet count were associated with bleeding episodes (supplementary appendix Table 3).

Thrombotic complications

Six patients had a thrombotic complication (Table 3). These patients were younger and appeared more severely ill. Three patients had a deep venous thrombosis (one associated with a peripherally inserted central catheter), one had superficial thrombophlebitis, one developed thrombosis-associated ischemic bowel, and one developed both portal vein and hepatic artery thrombosis (possibly related to heparin induced thrombocytopenia occurring after ELT). Only this last patient died as a result of their thrombotic complication. The median time from ICU admission to thrombotic complication was 6.5 days (IQR 5-9). In three of the cases, the thrombotic complication occurred after ELT, with at least four days between surgery and the thrombosis in each instance. Only a single patient with a thrombotic complication actually received blood products in the preceding 48 hours (prior to development of a deep venous thrombosis in this case). Comparisons of clotting parameters was not informative (supplementary appendix Table 4).

\section{Discussion}

Key Findings

In patients with severe ALF admitted to liver transplant ICUs in ANZ, we found that INR appeared to dominate coagulation monitoring and treatment, however, its association with bleeding was not significant. Low platelet counts and low fibrinogen concentrations were significantly associated with bleeding, however fibrinogen 
monitoring was significantly less frequent. Clotting factor use and blood product administration was common. However, unlike for other products, which differed in use according to clear derangements, the initial use of FFP did not. Bleeding occurred in less than a fifth of patients while thrombotic complications occurred in less than $10 \%$ and half occurred many days after ELT.

\section{Relationship to previous studies and current guidelines}

The dominant approach to hematological monitoring in ALF has been to focus on changes in INR or $\mathrm{PT}^{4,9-12}$. Our findings show this strategy has important limitations ${ }^{13,14}$, ${ }^{15}$; fibrinogen concentration and platelet count seems more relevant to the risk of bleeding. This insight into the relationship between fibrinogen and bleeding appears novel $^{14,16-24}$ (see supplementary appendix Table 5). Furthermore, apart from one study that evaluated registry data ${ }^{25}$, almost all such previous studies were single center in nature, with numbers similar to ours and often focused on very specific hematological parameters. Consistent with our findings, such studies found that INR improved over a short period of time in $\mathrm{ICU}^{26}$ and that bleeding was neither especially common nor clinically serious for the majority of patients ${ }^{25}$. In this regard, the pathophysiology of hemostasis in ALF is complex ${ }^{5}$ with preserved clotting capability ${ }^{17}$ and even hypercoagulability ${ }^{27}$ and, apart from case reports ${ }^{28}$, our study is only the second to systematically evaluate for thrombotic complications in patients with ALF.

Our findings suggest a somewhat liberal use of FFP despite lack of evidence ${ }^{29,30}$ or support by guidelines ${ }^{31-35}$. Unnecessary FFP administration is not without risk and its use may result in complications such as transfusion related acute lung injury, volume overload and even exacerbation of bleeding due to venous distension.

The aPTT is a measure of the intrinsic pathway but its usefulness as an outcome predictive value has been questioned ${ }^{32,33}$. Our findings raise the possibility that the aPTT may warrant re-evaluation. Thrombocytopenia is clearly associated with a bleeding tendency in liver patients ${ }^{25}, 34,35$. However, the association between hypofibrinogenemia and hemorrhage (as shown in our study) has not been previously 
described. Our findings suggest that monitoring fibrinogen concentrations and correcting very low levels may be desirable. PCC use was relatively uncommon in our study. In two small single center studies that evaluated a combined total of 33 ALF patients $^{36,37}$, PCC administration was shown to improve hemostatic parameters with a low incidence of thrombotic complications. Given the theoretical prothrombotic risks of $\mathrm{PCC}^{37-39}$ and the limited data on safety for use in ALF, further evaluation is warranted $^{40}$. Nonetheless, studies in liver transplantation suggest a favorable safety profile $^{41-43}$ and our study also found no association with thrombosis. Descriptions of rFVIla utilization in ALF patients have been reported in small retrospective single center observational studies ${ }^{16,44}$ however its cost is high and its safety is unclear. In our cohort, use of rFVIla was rare, but free of thrombotic complications.

Implications of study findings

Our findings imply that routine hemostatic parameters focus on INR but that the platelet count and the less frequently measured fibrinogen concentration may be more relevant to bleeding risk. Our findings also imply that, on average, maintaining a fibrinogen concentration $>1.5 \mathrm{~g} / \mathrm{L}$ and a platelet count $>75 \times 10^{9} / \mathrm{L}$ (both at the lower 95\% $\mathrm{Cl}$ for non-bleeding patients during their ICU admission) might represent a reasonable balance between bleeding and thrombosis risk in ALF, especially if bleeding has already occurred. Whilst the minimum safe level of these parameters currently remains unknown, these values are similar to current guidelines for ALF patients with a bleeding complication ${ }^{45}$. Moreover, our findings imply that, in the absence of the impact of surgery (e.g. ELT), thrombosis risk is markedly less than bleeding risk and not apparently related to clotting factor therapy.

\section{Strengths and limitations}

Our study has several strengths. All liver transplantation ANZ ICUs contributed to this study, thus providing the first assessment of binational ANZ practice. Complete laboratory and clinically relevant data were obtained. All data were obtained by experienced clinical researchers from patient records at each center, using a consistent methodology to ensure reliability of approach. We found important variability and 
shortcomings in the monitoring of fibrinogen, an observation with likely practical clinical implications. Finally, we report data on thrombosis for the first time.

We acknowledge some limitations. Although this study included ALF patients from every transplant unit in ANZ, only a relatively small number were evaluated. Some complications (such as thrombosis) affected only a small proportion of patients, limiting our analysis. However, ALF is uncommon, and our cohort is the largest of its type in the last 40 years and only the second ever to assess thrombotic events. The use of a convenience-based approach may have failed to provide an accurate overall representation of ALF patients. However, contributing units identified ten or more consecutive patients based solely on each of them fulfilling the primary diagnosis of ALF at the time of admission to ICU, thus limiting selection bias. We used a medical record based, retrospective approach. The accuracy of clinical data and investigation results are sometimes uncertain and treating clinicians may make errors during the entry of information into the patient record. However, all patients were treated in major ICUs with processes for complex data collection. Additionally, all submitted data was carefully checked prior to analysis. Finally, we did not collect data on other potentially useful measures of hemostasis, such as individual clotting factor concentrations or results of thromboelastography. These measures of hemostasis are not currently in wide-spread routine use, but thromboelastography in particular may have considerable utility as a convenient point of care functional test of hemostasis. Further studies are required to establish its role in guiding the management of ALF.

\section{Conclusion}

This is the largest study of coagulation testing and management, bleeding complications, thrombotic complications and associated management for ALF in the last 40 years. We found that fibrinogen was measured less often than other parameters, that the INR had a modest and non-significant association with bleeding while platelet count and fibrinogen levels did, thus suggesting that avoidance of extreme thrombocytopenia and hyperfibrinogenemia is important. There was no clear 
association between clotting factor administration or hemostatic parameters and thrombotic complications, further suggesting that correcting low fibrinogen and platelet levels is likely safe. Overall, these findings provide considerable baseline information for the design and powering of interventional trials and by demonstrating considerable practice variation help identify what interventions fall within the range of current practice and, therefore, could be ethically compared.

This article is protected by copyright. All rights reserved. 
Table 1. Intensity of coagulation and hematological testing during the first week in ICU

Testing data collected at $0,6,12,24$ hours and then daily to a total of 7 days INR= international normalized ratio, aPTT= activated partial thromboplastin time † comparisons with frequency of INR testing

This article is protected by copyright. All rights reserved. 
Table 2. Clinical Characteristics of ALF patients with and without bleeding episodes

Data presented as $\mathrm{n}(\%)$, median [IQR\}

APACHE III= acute physiology and chronic health evaluation III, KCC= Kings College Criteria, INR= international normalized ratio, aPTT= activated partial thromboplastin time, FFP= fresh frozen plasma, $\mathrm{rFVIla}=$ recombinant factor VII activated, ELT= emergency liver transplant

This article is protected by copyright. All rights reserved. 
Table 3. Characteristics of ALF patients with and without thrombotic complications

Data presented as $\mathrm{n}(\%)$ and median [IQR\}

APACHE III= acute physiology and chronic health evaluation III, KCC= Kings College Criteria, INR= international normalized ratio, aPTT= activated partial thromboplastin time, FFP= fresh frozen plasma, $\mathrm{rFVIla}=$ recombinant factor VII activated, ELT= emergency liver transplant

This article is protected by copyright. All rights reserved. 
Figure 1. Changes in hemostatic parameters over time in all ALF patients

Geometric means of log transformed data Error bars indicate $95 \% \mathrm{Cl}$ (only positive or negative values displayed for upper and lower lines respectively to assist visual clarity)

This article is protected by copyright. All rights reserved. 
Figure 2. Comparison of Fibrinogen concentration by bleeding episode status

Geometric means of log transformed data

Error bars indicate $95 \% \mathrm{Cl}$

$p=0.0004$ (value for overall differences between groups)

This article is protected by copyright. All rights reserved. 


\section{Figure 3. Comparison of platelet count by bleeding episode status}

Geometric means of log transformed data

Error bars indicate $95 \% \mathrm{Cl}$

$p<0.0001$ (value for overall differences between groups)

This article is protected by copyright. All rights reserved. 
Appendix of supplementary material

This article is protected by copyright. All rights reserved. 


\section{Supplementary Table 1. Patient characteristics, etiology and outcomes of ALF}

Data presented as median [interquartile range] and \% (n)

$\mathrm{HBV}=$ hepatitis $\mathrm{B}$ virus, $\mathrm{HT}=$ hypertension, $\mathrm{IHD}=$ ischemic heart disease, $\mathrm{HCV}=$ hepatitis $\mathrm{C}$ virus, ELT = emergency liver transplantation, $\mathrm{OD}=$ overdose, $\mathrm{HLH}=$ hemophagocytic lymphohistiocytosis, $\mathrm{BCS}=$ Budd-Chiari Syndrome, AFLP = acute fatty liver of pregnancy, PV = portal vein, KCC= Kings College Criteria, APACHE III= Acute Physiology and Chronic Health Evaluation III, ELT= emergency liver transplant

†Drugs involved include: buprenorphine, agomelatine, infliximab, methylenedioxymethamphetamine ('ecstasy' or MDMA) and methamphetamines.

This article is protected by copyright. All rights reserved. 


\section{Supplementary Figure 1. Comparison of international normalized ratio on basis of bleeding}

INR=international normalized ratio

Geometric means of log transformed data

Error bars indicate $95 \% \mathrm{Cl}$

$p=0.02$ (value for overall differences between groups)

This article is protected by copyright. All rights reserved. 
Supplementary Figure 2. Comparison of activated partial thromboplastin time on basis of bleeding

aPTT=activate partial thromboplastin time

Geometric means of log transformed data

Error bars indicate $95 \% \mathrm{Cl}$

$p=0.15$ (value for overall differences between groups)

This article is protected by copyright. All rights reserved. 
Supplementary Figure 3. International Normalized Ratio and Fresh Frozen Plasma use

$\mathrm{FFP}=$ fresh frozen plasma, INR= international normalized ratio

This article is protected by copyright. All rights reserved. 


\section{Supplementary Figure 4. Fibrinogen concentration and cryoprecipitate use}

This article is protected by copyright. All rights reserved. 
Supplementary Figure 5. Platelet count and platelet transfusion use

This article is protected by copyright. All rights reserved. 


\section{Supplementary Table 2. Details of twelve bleeding patients}

Five bleeding patients were investigated with a computerized tomography scan, two were evaluated with ultrasound and five had no imagining performed. All bleeding patients received blood products at the time of their bleeding episode (weekly total summarized in Table 3). At the time of bleeding, most (8/12) patients were managed with a combination of PRBC, FFP, cryoprecipitate and platelets, while a minority $(3 / 12)$ of patients were managed with just a platelet transfusion with or without cryoprecipitate. A single patient received FFP, cryoprecipitate and PCC but no platelets. For those patients who died, bleeding was implicated as a direct contributing factor in only three patients (two ICH's and one spontaneous intra-abdominal bleed). The hemostatic profile of both $\mathrm{ICH}$ patients was extremely deranged at the time of the event (INR of 7.6 and 15, aPTT of 87 and $57 \mathrm{sec}$, fibrinogen of 0.2 and $0.6 \mathrm{~g} / \mathrm{L}$ and platelet count of $31 \times 10^{9} / \mathrm{L}$ and $114 \times 10^{9} / \mathrm{L}$, respectively). For the other four patients who experienced a bleeding episode and subsequently died, a minimum of 62 hours elapsed between these events and none of them died in a manner directly attributed to hemorrhage.

This article is protected by copyright. All rights reserved. 
Supplementary Table 3. Hemostatic parameters in twelve bleeding patients at the time of documented hemorrhage, at a time without documented hemorrhage and compared to matched controls

INR= international normalized ratio, aPTT= activated partial thromboplastin time, $\mathrm{POD}=$ paracetamol overdose, $\mathrm{KCC}=\mathrm{Kings}$ College $\mathrm{Criteria}$ Data presented as median [IQR]

†bleeding patient as own control

łbleeding patient versus matched control

This article is protected by copyright. All rights reserved. 
Supplementary Table 4. Hemostatic parameters in six patients with a thrombotic complication of at the time thrombosis occurred compared to parameters measured four days previously

INR= international normalized ratio, aPTT= activated partial thromboplastin time

Data presented as median [IQR]

This article is protected by copyright. All rights reserved. 


\section{Supplementary Table 5. Studies of Hemostasis and Blood Product Utilization in Acute Liver Failure}

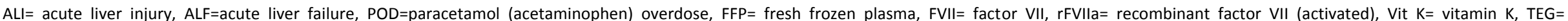
thromboelastography, $v W F=$ von Willebrand-Factor, $A K I=$ acute kidney injury, ARF= acute renal failure, INR= international normalized ratio

Routine clotting profile= standard laboratory assessment of hemostasis utilized for most clinical care; includes INR, aPTT, platelet count and fibrinogen concentration

Extended hemostatic parameters= Complex hematological testing additional to routine clotting profile; includes individual factor concentrations, factor activity, thromboelastography, fibrinogen degradation products as well as standard clotting profile 


\section{References}

[1] Warrillow S, Bailey M, Pilcher D, et al. Characteristics and Outcomes of Patients with Acute Liver Failure Admitted to Australian and New Zealand Intensive Care Units. Internal medicine journal. 2018.

[2] Warrillow S, Tibballs $\mathrm{H}$, Bailey $\mathrm{M}$, et al. Characteristics, management and outcomes of patients with acute liver failure admitted to Australasian intensive care units. Crit Care Resusc. 2019; 21: 188-99.

[3] Warrillow S, Bellomo R. Preventing cerebral oedema in acute liver failure: the case for quadruple-H therapy. Anaesth Intensive Care. 2014; 42: 78-88.

[4] Wlodzimirow KA, Eslami S, Abu-Hanna A, Nieuwoudt M, Chamuleau RA. Systematic review: acute liver failure - one disease, more than 40 definitions. Aliment Pharmacol Ther. 2012; 35: 1245-56.

[5] Lisman T, Porte RJ. Rebalanced hemostasis in patients with liver disease: evidence and clinical consequences. Blood. 2010; 116: 878-85.

[6] Lee $\mathrm{KCL}$, Baker L, Mallett $\mathrm{S}$, et al. Hypercoagulability progresses to hypocoagulability during evolution of acetaminophen-induced acute liver injury in pigs. Scientific Reports. 2017; 7: 9347.

[7] Northup PG. Hypercoagulation in liver disease. Clin Liver Dis. 2009; 13: 109-16.

[8] von Elm E, Altman DG, Egger $M$, et al. The Strengthening the Reporting of Observational Studies in Epidemiology (STROBE) statement: guidelines for reporting observational studies. Lancet. 2007; 370: 1453-7.

[9] Wlodzimirow KA, Eslami S, Chamuleau RA, Nieuwoudt M, Abu-Hanna A. Prediction of poor outcome in patients with acute liver failure-systematic review of prediction models. PloS one. 2012; 7: e50952.

[10] Cardoso FS, Karvellas CJ. Managing the patient with acute liver failure. Clinical liver disease. 2017; 9: 89-93.

[11] Patton $H$, Misel $M$, Gish RG. Acute liver failure in adults: an evidence-based management protocol for clinicians. Gastroenterol Hepatol ( $N$ Y). 2012; 8: 161-212.

[12] O'Grady JG, Alexander GJ, Hayllar KM, Williams R. Early indicators of prognosis in fulminant hepatic failure. Gastroenterology. 1989; 97: 439-45.

[13] Harrison MF. The Misunderstood Coagulopathy of Liver Disease: A Review for the Acute Setting. The western journal of emergency medicine. 2018; 19: 863-71.

[14] Agarwal B, Wright G, Gatt A, et al. Evaluation of coagulation abnormalities in acute liver failure. Journal of Hepatology. 2012; 57: 780-6.

[15] Stravitz RT, Ellerbe C, Durkalski V, Reuben A, Lisman T, Lee WM. Thrombocytopenia Is Associated With Multi-organ System Failure in Patients With Acute Liver Failure. Clinical gastroenterology and hepatology : the official clinical practice journal of the American Gastroenterological Association. 2016; 14: 613-20.e4.

[16] Shami VM, Caldwell SH, Hespenheide EE, Arseneau KO, Bickston SJ, Macik BG. Recombinant activated factor VII for coagulopathy in fulminant hepatic failure compared with conventional therapy. Liver Transpl. 2003; 9: 138-43.

[17] Stravitz RT, Lisman T, Luketic VA, et al. Minimal effects of acute liver injury/acute liver failure on hemostasis as assessed by thromboelastography. Journal of hepatology. 2012; 56: 129-36. 
[18] Habib M, Roberts LN, Patel RK, Wendon J, Bernal W, Arya R. Evidence of rebalanced coagulation in acute liver injury and acute liver failure as measured by thrombin generation. Liver International. 2014; 34: 672-8.

[19] Hugenholtz GC, Adelmeijer J, Meijers JC, Porte RJ, Stravitz RT, Lisman T. An unbalance between von Willebrand factor and ADAMTS13 in acute liver failure: implications for hemostasis and clinical outcome. Hepatology. 2013; 58: 752-61.

[20] Lisman T, Bakhtiari K, Adelmeijer J, Meijers JC, Porte RJ, Stravitz RT. Intact thrombin generation and decreased fibrinolytic capacity in patients with acute liver injury or acute liver failure. Journal of thrombosis and haemostasis : JTH. 2012; 10: 1312-9.

[21] Agarwal B, Gatt A, Riddell A, et al. Hemostasis in patients with acute kidney injury secondary to acute liver failure. Kidney international. 2013; 84: 158-63.

[22] Stravitz RT, Bowling R, Bradford RL, et al. Role of procoagulant microparticles in mediating complications and outcome of acute liver injury/acute liver failure. Hepatology. 2013; 58: 304-13.

[23] Gazzard BG, Henderson JM, Williams R. Early changes in coagulation following a paracetamol overdose and a controlled trial of fresh frozen plasma therapy. Gut. 1975; 16: 617-20.

[24] Gazzard BG, Henderson JM, Williams R. Factor VII levels as a guide to prognosis in fulminant hepatic failure. Gut. 1976; 17: 489-91.

[25] Stravitz RT, Ellerbe C, Durkalski V, et al. Bleeding complications in acute liver failure. Hepatology. 2018; 67: 1931-42.

[26] Pereira SP, Rowbotham D, Fitt S, Shearer MJ, Wendon J, Williams R. Pharmacokinetics and efficacy of oral versus intravenous mixed-micellar phylloquinone (vitamin K1) in severe acute liver disease. J Hepatol. 2005; 42: 365-70.

[27] Allison MG, Shanholtz CB, Sachdeva A. Hematological Issues in Liver Disease. Critical Care Clinics. 2016; 32: 385-96.

[28] Scarlatescu E, Tomescu DR. Prothrombotic State in a Patient With Acute Liver Failure: The Question of Anticoagulation. Seminars in cardiothoracic and vascular anesthesia. 2018; 22: 174-9.

[29] Lisman T, Caldwell SH, Burroughs AK, et al. Hemostasis and thrombosis in patients with liver disease: the ups and downs. J Hepatol. 2010; 53: 362-71.

[30] De Gasperi A, Corti A, Mazza E, Prosperi M, Amici O, Bettinelli L. Acute liver failure: managing coagulopathy and the bleeding diathesis. Transplant Proc. 2009; 41: 1256-9.

[31] Escudié L, Francoz C, Vinel J-P, et al. Amanita phalloides poisoning: Reassessment of prognostic factors and indications for emergency liver transplantation. Journal of Hepatology. 46: 466-73.

[32] Kim T, Lee $\mathrm{D}$, Lee $\mathrm{JH}$, et al. Predictors of poor outcomes in patients with wild mushroom-induced acute liver injury. World J Gastroenterol. 2017; 23: 1262-7.

[33] Blasi A. Coagulopathy in liver disease: Lack of an assessment tool. World journal of gastroenterology. 2015; 21: 10062-71.

[34] Fisher NC, Mutimer DJ. Central venous cannulation in patients with liver disease and coagulopathy--a prospective audit. Intensive Care Med. 1999; 25: 481-5.

[35] Northup PG, Caldwell SH. Coagulation in liver disease: a guide for the clinician. Clinical gastroenterology and hepatology : the official clinical practice journal of the American Gastroenterological Association. 2013; 11: 1064-74. 
[36] Drebes A, de Vos M, Gill S, et al. Prothrombin Complex Concentrates for Coagulopathy in Liver Disease: Single-Center, Clinical Experience in 105 Patients. Hepatology communications. 2019; 3: 513-24.

[37] Blais N, Rioux-Masse B. Use Of Prothrombin Complex Concentrates In Patients With Hepatic Coagulopathy: A Single Center Retrospective. Blood. 2013; 122: 2400.

[38] Sørensen B, Spahn DR, Innerhofer P, Spannagl M, Rossaint R. Clinical review: Prothrombin complex concentrates - evaluation of safety and thrombogenicity. Critical Care. 2011; 15: 201.

[39] Tischendorf $M$, Fuchs $A$, Zeuzem S, Lange CM. Use of prothrombin complex concentrates in patients with decompensated liver cirrhosis is associated with thromboembolic events. Journal of Hepatology. 2019; 70: 800-1.

[40] Kujovich JL. Coagulopathy in liver disease: a balancing act. Hematology American Society of Hematology Education Program. 2015; 2015: 243-9.

[41] Kirchner C, Dirkmann D, Treckmann JW, et al. Coagulation management with factor concentrates in liver transplantation: a single-center experience. Transfusion. 2014; 54: 2760-8.

[42] Dötsch TM, Dirkmann D, Bezinover D, et al. Assessment of standard laboratory tests and rotational thromboelastometry for the prediction of postoperative bleeding in liver transplantation. BJA: British Journal of Anaesthesia. 2017; 119: 402-10.

[43] Hartmann M, Walde C, Dirkmann D, Saner FH. Safety of coagulation factor concentrates guided by ROTEM ${ }^{\mathrm{TM}}$-analyses in liver transplantation: results from 372 procedures. BMC anesthesiology. 2019; 19: 97.

[44] KrisI JC, Meadows HE, Greenberg CS, Mazur JE. Clinical usefulness of recombinant activated factor VII in patients with liver failure undergoing invasive procedures. Ann Pharmacother. 2011; 45: 1433-8.

[45] Wendon J, Cordoba J, Dhawan A, et al. EASL Clinical Practical Guidelines on the management of acute (fulminant) liver failure. J Hepatol. 2017; 66: 1047-81.

[46] Dymock IW, Tucker JS, Woolf IL, Poller L, Thomson JM. Coagulation studies as a prognostic index in acute liver failure. British journal of haematology. 1975; 29: 385-95.

[47] Schiodt FV, Balko J, Schilsky M, Harrison ME, Thornton A, Lee WM. Thrombopoietin in acute liver failure. Hepatology. 2003; 37: 558-61.

This article is protected by copyright. All rights reserved. 


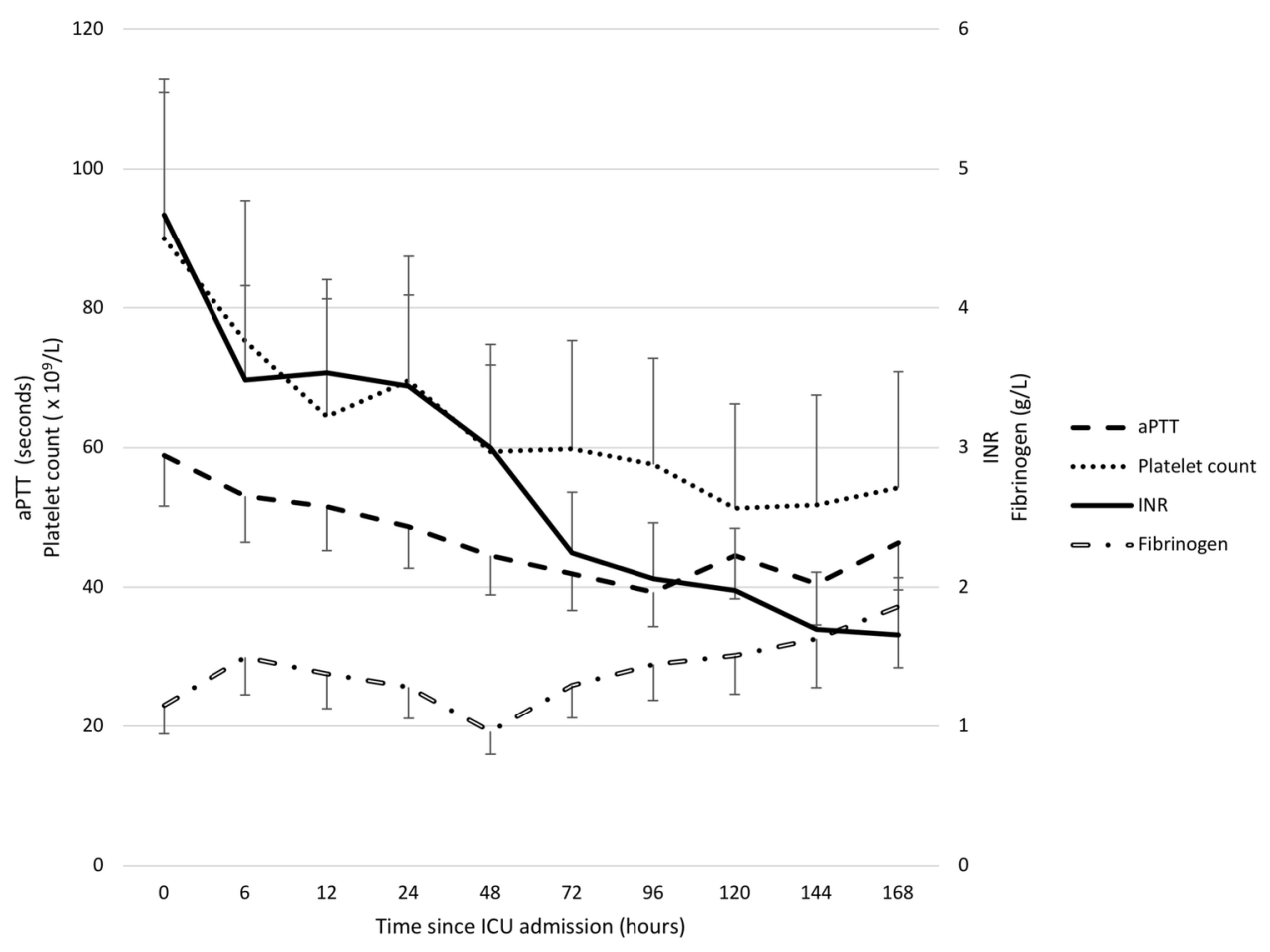

JGH_14876_Figure1.tiff

This article is protected by copyright. All rights reserved. 


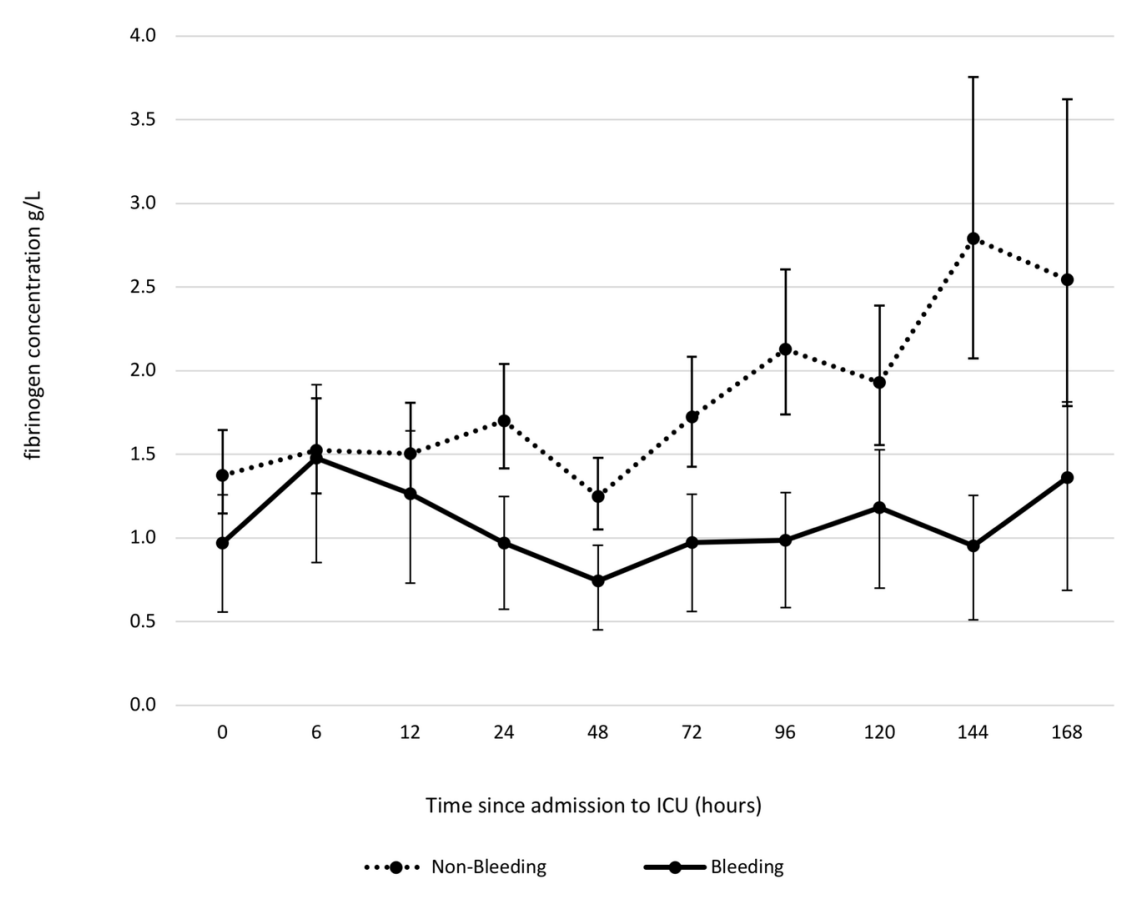

JGH_14876_Figure 2.tiff

This article is protected by copyright. All rights reserved. 


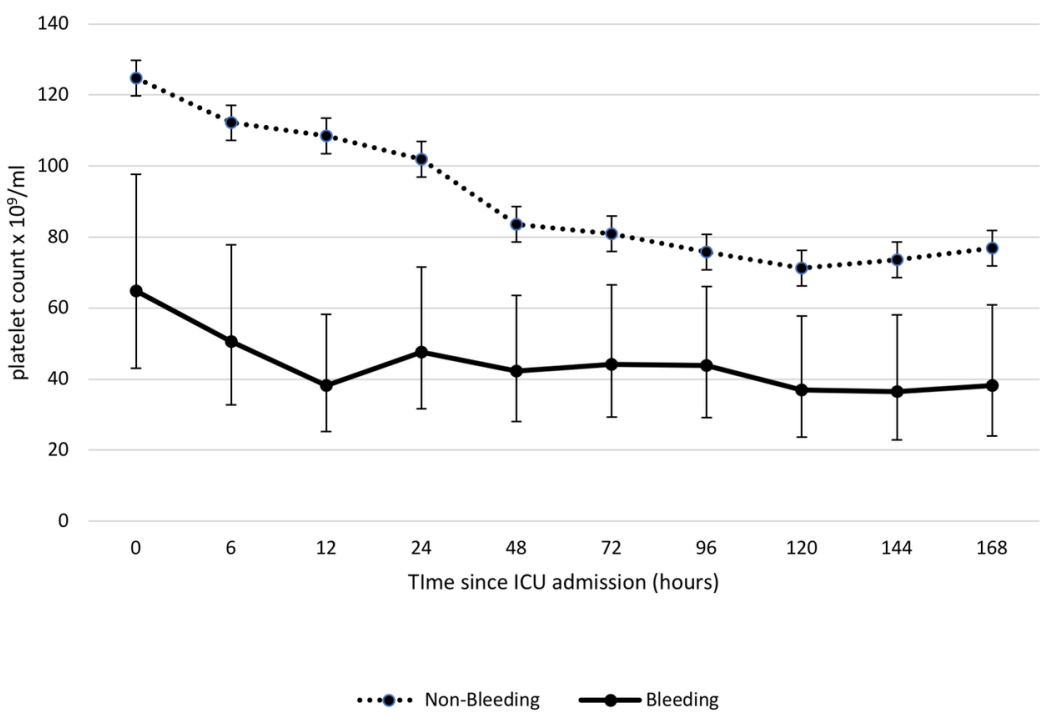

JGH_14876_Figure 3.tiff

This article is protected by copyright. All rights reserved. 


\section{University Library}

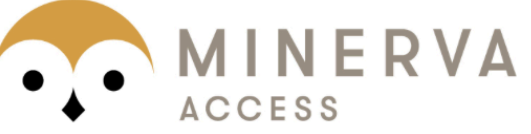

A gateway to Melbourne's research publications

Minerva Access is the Institutional Repository of The University of Melbourne

Author/s:

Warrillow, S;Fisher, C;Tibballs, H;Bailey, M;McArthur, C;Lawson-Smith, P;Prasad, B;Anstey, M;Venkatesh, B;Dashwood, G;Walsham, J;Holt, A;Wiersema, U;Gattas, D;Zoeller, M;Garcia Alvarez, M;Bellomo, $\mathrm{R}$

Title:

Coagulation abnormalities, bleeding, thrombosis, and management of patients with acute liver failure in Australia and New Zealand

Date:

2019-11-05

Citation:

Warrillow, S., Fisher, C., Tibballs, H., Bailey, M., McArthur, C., Lawson-Smith, P., Prasad, B., Anstey, M., Venkatesh, B., Dashwood, G., Walsham, J., Holt, A., Wiersema, U., Gattas, D., Zoeller, M., Garcia Alvarez, M. \& Bellomo, R. (2019). Coagulation abnormalities, bleeding, thrombosis, and management of patients with acute liver failure in Australia and New Zealand. JOURNAL OF GASTROENTEROLOGY AND HEPATOLOGY, 35 (5), pp.846-854. https://doi.org/10.1111/jgh.14876.

Persistent Link:

http://hdl.handle.net/11343/286579 